\title{
Hodentumoren: chronische Fatigue nach der kurativen Therapie
}

\begin{abstract}
Langzeitüberlebende eines Hodentumors entwickeln häufiger eine chronische Fatigue als Männer, die nicht an dieser malignen Erkrankung litten. In einer explorativen Studie wurden mögliche Risikofaktoren median 12 und 19 Jahre nach der erfolgreichen Krebstherapie untersucht.
\end{abstract}

$C$ hrou hronische Fatigue (CF) zählt zu den häufigen unerwünschten Begleiterscheinungen einer Krebserkrankung und -therapie. Sie beeinträchtigt die Lebensqualität in besonderem Maße. Es gibt nur wenige bevölkerungsbasierte und longitudinale Studien, die sich mit der Entwicklung einer CF, möglichen Risikofaktoren und der Langzeitprävalenz befassen. In Norwegen betragen die relativen 5-Jahres-Überlebensraten für die meist jungen Patienten mit Hodentumoren über $97 \%$. Die Gruppe ist daher prädestiniert für Studien zum „Cancer-Survivorship“ mit Schwerpunkt „therapiebedingte Langzeitwirkungen“.

In einer explorativen Studie mit Fokus auf der CF-Prävalenz wurde eine bevöl- kerungsbasierte Kohorte median 12 (SI, 1998-2002) und 19 Jahre (SII, 20072008) nach erfolgreicher Therapie eines Keimzelltumors untersucht. Für 812 zwischen 1980-1994 behandelte Männer lagen Werte für Testosteron und luteinisierendes Hormon (LH) vor. In den Untersuchungszeiträumen SI und SII beantworteten die Patienten Fragebögen zu Fatigue, Neurotoxizität, Angst und Depression und machten Angaben zu Komorbiditäten, Medikamenteneinnahme, Tabakkonsum und Ehestatus.

Die CF-Prävalenz erhöhte sich vom Untersuchungszeitraum SI zu SII von 15 auf $27 \%$. In letzterem Zeitraum war das CF-Risiko für Männer mit hochgradiger Neuropathie 3- bis 4-fach erhöht. Auch ein erniedrigter Testosteronspiegel begünstigte die Entwicklung einer $\mathrm{CF}$, ebenso hohe Werte auf der Angst- und Depressionsskala. Moderate und hohe körperliche Aktivität wirkten hingegen protektiv.

Fazit: Langfristig erhöht sich die Prävalenz einer CF bei kurativ behandelten Hodentumorpatienten. Eine frühzeitige Behandlung von Angst- und Depressionssymptomen und möglicherweise eine Testosteronsubstitution könnten das Risiko reduzieren. Wolfgang Zimmermann

Sprauten M et al. Chronic fatigue in 812 testicular cancer survivors during long-term follow up: increasing prevalence and risk factors. Ann Oncol. 2015;26(10):2133-40.

\section{RCC: mTOR-Inhibitor nach gescheiterter Anti-VEGF-Therapie}

\begin{abstract}
Progressionsfreie und Gesamtüberlebenszeit von Patienten mit metastasiertem Nierenzellkarzinom lassen sich nach dem Scheitern von Anti-VEGF-Therapien durch Everolimus verbessern. Jetzt gibt es neue Daten zu Sicherheit und Wirksamkeit dieser Intervention.
\end{abstract}

\footnotetext{
eitlinien empfehlen zur ErstlinientheLapie des metastasierten klarzelligen Nierenzellkarzinoms (mRCC) bei günstigem MSKCC-Risikoscore die VEGF (,vascular endothelial growth factor")Rezeptortyrosinkinaseinhibitoren Sunitinib, Pazopanib oder den VEGF-Antikörper Bevacizumab. Eine Zweitlinientherapie mit dem mTOR-Inhibitor Everolimus verbesserte in früheren Studien gegenüber Placebo signifikant das progressionsfreie Überleben.

Ein Team um Laurence Albiges präsentierte nun die gepoolten Daten von vier prospektiven, nicht interventionellen Studien zu Wirksamkeit und Sicherheit von Everolimus bei Patienten mit mRCC,
}

die nach einer oder zwei Anti-VEGF-Therapien einen Progress erlitten hatten. Von den 632 Patienten hatten die meisten einen günstigen oder intermediären MSKCC-Risikoscore, einen klarzelligen Tumor und eine Nephrektomie erhalten. Häufigste Ersttherapie war Sunitinib, 493 Patienten erhielten eine Zweitlinientherapie mit Everolimus, 48 Patienten eine Drittlinien- und 19 weitere Therapien. Die Behandlung wurde fortgesetzt bis zum Krankheitsprogress oder dem Auftreten inakzeptabler Nebenwirkungen.

Als mediane Zeit bis zur Progression ermittelten die Forscher 6,3 Monate für alle Patienten und 6,4 Monate für die in der Zweitlinie mit Everolimus Behandel- ten. Die entsprechenden medianen Zeiten für das progressionsfreie Überleben: 5,5 bzw. 5,8 Monate. $12 \%$ erreichten eine komplette oder partielle Remission, $59 \%$ eine Krankheitsstabilisierung. Das mediane Gesamtüberleben lag bei 11,2 Monaten. Als unerwünschte Ereignisse traten am häufigsten Stomatitis (25\%), Anämie (15\%) und Asthenie (11\%) auf.

Fazit: Die Ergebnisse dieser gepoolten Analyse belegen Sicherheit und Wirksamkeit von Everolimus als Zweitlinientherapie und stützen den Einsatz des mTOR-Inhibitors als eine Standardtherapie für Patienten mit einem unter AntiVEGF-Therapie progredienten Nierenzellkarzinom. Wolfgang Zimmermann

Albiges $L$ et al. Everolimus for patients with metastatic renal cell carcinoma refractory to anti-VEGF therapy: results of a pooled analysis of non-interventional studies. Eur J Cancer. 2015;51(16):2368-74. 